\title{
Diffusion and Brownian Motion: On the Possibility to Control Diffusion of Small Particles with Laser Radiation
}

\author{
A.A. Afanas'ev ${ }^{a}$, Yu.A. Kurochkin ${ }^{a}$, S.Yu. Mikhnevich ${ }^{b}$ And D.V. Novitsky ${ }^{a, *}$ \\ ${ }^{a}$ B.I. Stepanov Institute of Physics, National Academy of Sciences of Belarus, \\ Nezavisimosti Av. 68, 220072 Minsk, Belarus \\ ${ }^{b}$ Belarusian State University of Informatics and Radioelectronics, P. Brovki Str. 6, 220013 Minsk, Belarus
}

(Received July 6, 2017; in final form January 10, 2018)

In this paper, we discuss several problems for the diffusion equation taking into account an external force (the Smoluchowski equation). Using the fundamental solution of the free diffusion equation, the Smoluchowski equation is solved in the presence of the small gradient force induced by an interference field of two laser beams. The fundamental solution is obtained for the diffusion equation in the presence of a constant external force, which is a gradient of a linear potential; this equation describes the process of distribution over the whole volume of particles initially concentrated in a small spatial domain. We study kinetics of the concentration gratings of transparent microspheres in a liquid induced by the gradient force in the interference field of laser radiation.

DOI: 10.12693/APhysPolA.135.326

PACS/topics: 05.40.Jc, 42.62.-b, 42.25.Fx, 42.65.Hw

\section{Introduction}

Manipulation of small particle motion and localization with the light pressure forces generated by laser radiation is currently one of the most interesting problems lying at the interfaces between electrodynamics, optics, mechanics, and quantum electronics. The relevance of this problem is mainly due to many possible applications, especially in medicine and biology. Thus, the apparatus for laser transportation and confinement of dielectric particles was first demonstrated in 1986 [1, 2]. A year later, using a beam of IR laser radiation, the same group succeeded in transportation of a living cell without any damage [3]. The device, which gives the possibility of nondestructive transportation of small dielectric particles and biological objects with laser radiation, was called "laser tweezers". At present, laser tweezers are widely used in biology and medicine for study of viruses and bacteria [4], DNA molecules [5], processes inside living cells [6], etc.

Apart from biological and medical applications, light pressure can be used to write concentration gratings in liquid suspensions of dielectric particles with controllable optical properties. Such artificial heterogeneous media are shown to have large values of optical Kerr coefficient and can find applications as wideband nonlinear media for laser radiation of low intensity and large pulse duration $[7,8]$.

\section{The main equation}

To study the processes of displacement and localization of small particles, one can use the diffusion equation that takes into account the presence of an external force (the Smoluchowski equation). When an external field is

\footnotetext{
*corresponding author; e-mail: dvnovitsky@gmail.com
}

imposed, the change in particle velocity is proportional to the force, with a proportionality coefficient $b$, which is called particle mobility [9-11]. Then, the diffusion equation with a term for the external force $F_{i}$ can be written as follows:

$$
\frac{\partial n(x, t)}{\partial t}=D \frac{\partial^{2} n(x, t)}{\partial x_{i}^{2}}-\frac{\partial\left[n(x, t) b F_{i}\right]}{\partial x_{i}} .
$$

Here $n(x, t)=N f(x, t)$ is the concentration of particles, $f(x, t)$ - the distribution function, $D$ - the diffusion coefficient, and $N$ - the total number of particles. Equation (1) can be regarded as the conservation law for the number of suspended particles in a differential form

$$
\frac{\partial n(x, t)}{\partial t}=-\frac{\partial j_{i}(x, t)}{\partial x_{i}},
$$

where $j_{i}(x, t)=N J_{i}(x, t)$ is the particle flux density $(i=1,2,3)$.

In general, the existence of a flux of diffusing particles can also be due to the presence of temperature and pressure gradients [8]. However, since the medium is assumed to be in thermodynamic equilibrium, the temperature gradient is absent. The pressure gradient can be caused, for example, by an external force $F_{i}$. In the presence of external force, the expression for the particle flux density takes the form [9]:

$$
j_{i}(x)=-D \frac{\partial n(x, t)}{\partial x_{i}}+n(x, t) b F_{i} .
$$

Here we take into account that the particle flux density is the product of the particle density by the velocity. The integral form of the conservation law for the number of particles is

$$
\int n(x, t) \mathrm{d}^{3} x=N \int f(x, t) \mathrm{d}^{3} x=1 .
$$

Note that the force should be a slow function of coordinates, i.e. the linear dimensions of the region of force variation should significantly exceed the mean free path 
of diffusing particles [10]. In Refs. [10, 12], the derivation of the diffusion equation is given on the basis of stochastic models. This, in particular, makes it possible to establish the connection between the diffusion equation and the telegraph equation. Further in this paper we use Eq. (1).

\section{Diffusion and Brownian motion}

A. Einstein derived the diffusion equation without taking into account the effect of force on particles of a substance dissolved in some liquid or a substance in the gaseous state introduced into another gas [13]. It was assumed that the physical cause for spreading the solute over the entire volume of the solvent is the Brownian motion, i.e. occasional kicks from the molecules of the solvent (or the gas into which the molecules of another gas were inserted) that the molecules of the solute or the particles of the suspension experience. As is known, A. Einstein and M. Smoluchowski, who used the integral equation, were the founders of the theory of Brownian motion as a theory of random processes [14].

The historical analysis of the influence of Smoluchowski's ideas on the development of kinetic theory as well as the origin of fundamental equations used in the mathematical description of stochastic processes is given in the work [15]. However, we would like to single out the Wiener contribution which have been developed in 1920 as the theory of functions describing the trajectories of Brownian particles. A characteristic feature of such functions is their continuity and non-differentiability in the entire domain of definition. However, one can calculate an integral for them. The construction of the integral for functions describing Brownian trajectories is called the Wiener measure and lies at the basis of the Feynman path integral formulation of quantum mechanics [16], as well as the basis of the quantum field theory formulation in terms of functional integration [17]. The connection between statistical physics and quantum theory in this latter approach is manifested in the fact that one can pass from quantum theory to statistical physics using the formal method called the Wick rotation of the real time axis in the complex plane by $\pi / 2$, which implies the transition to the imaginary time in the corresponding functionals of the quantum theory. The basis for such a procedure is the possibility of analytic continuation of some basic expressions used in quantum field theory to the complex domain. This possibility is one of the most important results of axiomatic quantum field theory.

\section{The fundamental solution of the diffusion equation}

Let us consider the redistribution over the whole volume for particles initially concentrated in a small volume near the point $\boldsymbol{x}_{0}$, when the process is affected by an external constant force. We should solve the modified Eq. (1) with a delta-like source

$$
\begin{aligned}
& \frac{\partial G\left(\boldsymbol{x}-\boldsymbol{x}_{0}, t-t_{0}\right)}{\partial t}-D \frac{\partial^{2} G\left(\boldsymbol{x}-\boldsymbol{x}_{0}, t-t_{0}\right)}{\partial \boldsymbol{x}^{2}} \\
& +\frac{\partial\left[G\left(\boldsymbol{x}-\boldsymbol{x}_{0}, t-t_{0}\right) b \boldsymbol{F}\right]}{\partial \boldsymbol{x}}=\delta\left(\boldsymbol{x}-\boldsymbol{x}_{0}\right) \delta\left(t-t_{0}\right) .
\end{aligned}
$$

To solve this equation, we use the standard method which emphasizes the closeness of the methods of diffusion theory and quantum mechanics. Application of this method to the diffusion equation has a certain methodological advantage related to the uniqueness of the solution choice, in contrast to the case of quantum mechanics. We represent the function $G$ and the delta functions in the righthand side of the equation in the form of the Fourier integrals

$$
\begin{aligned}
& G\left(\boldsymbol{x}-\boldsymbol{x}_{0}, t-t_{0}\right)= \\
& \quad \int G(\boldsymbol{k}, \omega) \exp \left(\mathrm{i}\left[\omega\left(t-t_{0}\right)-\boldsymbol{k}\left(\boldsymbol{x}-\boldsymbol{x}_{0}\right)\right]\right) \frac{\mathrm{d}^{3} k}{(2 \pi)^{3}} \frac{\mathrm{d} \omega}{2 \pi}, \\
& \delta\left(\boldsymbol{x}-\boldsymbol{x}_{0}\right) \delta\left(t-t_{0}\right)= \\
& \quad \int \exp \left(i\left[\omega\left(t-t_{0}\right)-\boldsymbol{k}\left(\boldsymbol{x}-\boldsymbol{x}_{0}\right)\right]\right) \frac{\mathrm{d}^{3} k}{(2 \pi)^{3}} \frac{\mathrm{d} \omega}{2 \pi} .
\end{aligned}
$$

Substituting these expressions into Eq. (5), we obtain the algebraic equation

$$
\left(\mathrm{i} \omega+D k^{2}-\mathrm{i} b \boldsymbol{k} \cdot \boldsymbol{F}\right) G(\boldsymbol{k}, \omega)=1,
$$

which results in

$$
G(\boldsymbol{k}, \omega)=\frac{1}{\mathrm{i} \omega+D k^{2}-\mathrm{i} b \boldsymbol{k} \cdot \boldsymbol{F}} .
$$

We substitute expression (9) into Eq. (6) and perform integration with respect to $\omega$. In order to do this, we continue $\omega$ to the upper part of the complex plane, i.e. $\omega$ is regarded as a complex variable with a positive imaginary part. This is possible, because the integrand in the expression

$$
\begin{aligned}
& G\left(\boldsymbol{x}-\boldsymbol{x}_{0}, t-t_{0}\right)= \\
& \quad \int \frac{-\mathrm{i} \exp \left(\mathrm{i}\left[\omega\left(t-t_{0}\right)-\boldsymbol{k}\left(\boldsymbol{x}-\boldsymbol{x}_{0}\right)\right]\right)}{\omega-\mathrm{i} D k^{2}+b \boldsymbol{k} \cdot \boldsymbol{F}} \frac{\mathrm{d}^{3} k}{(2 \pi)^{3}} \frac{\mathrm{d} \omega}{2 \pi}
\end{aligned}
$$

is an exponentially decreasing function, and therefore the line, along which the integration is performed and which initially coincides with the real axis in the complex $\omega$ plane, can be completed (closed) in the upper half-plane by a circle of infinitely large radius. The integral in this case will be equal to the pole residue at the point $\omega=\mathrm{i} D k^{2}-b \boldsymbol{k} \cdot \boldsymbol{F}$. The pole is in the upper half-plane, since $D$ is positive. Thus, after integration with respect to $\omega$, we have the integral

$$
\begin{aligned}
& G\left(\boldsymbol{x}-\boldsymbol{x}_{0}, t-t_{0}\right)= \\
& \quad \int \exp \left(\left(\mathrm{i} b \boldsymbol{k} \cdot \boldsymbol{F}-D k^{2}\right)\left(t-t_{0}\right)\right) \\
& \quad \times \exp \left(-\mathrm{i} \boldsymbol{k}\left(\boldsymbol{x}-\boldsymbol{x}_{0}\right)\right) \frac{\mathrm{d}^{3} k}{(2 \pi)^{3}},
\end{aligned}
$$

which is the three-dimensional Fourier transform of the function $\exp \left(\left(\mathrm{i} b \boldsymbol{k} \cdot \boldsymbol{F}-D k^{2}\right)\left(t-t_{0}\right)\right)$. This integral is easily converted to the standard one. Indeed, factorizing $D\left(t-t_{0}\right)$ and finding the perfect square in the exponent, we obtain 


$$
\begin{aligned}
& G\left(\boldsymbol{x}-\boldsymbol{x}_{0}, t-t_{0}\right)= \\
& \quad \exp \left(-\frac{b^{2} F^{2}\left(t-t_{0}\right)}{4 D}+\frac{b \boldsymbol{F} \cdot\left(\boldsymbol{x}-\boldsymbol{x}_{0}\right)}{2 D}\right) \\
& \quad \times \int \exp \left(-D k^{\prime 2}\left(t-t_{0}\right)\right) \exp \left(-\mathrm{i} \boldsymbol{k}^{\prime}\left(\boldsymbol{x}-\boldsymbol{x}_{0}\right)\right) \frac{\mathrm{d}^{3} k^{\prime}}{(2 \pi)^{3}},
\end{aligned}
$$

where $\boldsymbol{k}^{\prime}=\boldsymbol{k}-\mathrm{i} b \boldsymbol{F} / 2 D$ is the shifted argument. After integration we come to the expression

$$
\begin{aligned}
& G\left(\boldsymbol{x}-\boldsymbol{x}_{0}, t-t_{0}\right)=\frac{1}{\left[4 \pi D\left(t-t_{0}\right)\right]^{3 / 2}} \\
& \quad \times \exp \left(-\frac{b^{2} F^{2}\left(t-t_{0}\right)}{4 D}+\frac{b \boldsymbol{F} \cdot\left(\boldsymbol{x}-\boldsymbol{x}_{0}\right)}{2 D}-\frac{\left(\boldsymbol{x}-\boldsymbol{x}_{0}\right)^{2}}{4 D\left(t-t_{0}\right)}\right) .
\end{aligned}
$$

It is obvious that for $\boldsymbol{F}=0$, Eq. (13) as expected passes into the solution of the corresponding equation, not taking into account the presence of an external force.

Formula (13) can be rewritten in a compact form convenient for calculating the average values

$$
\begin{aligned}
& G\left(\boldsymbol{x}-\boldsymbol{x}_{0}, t-t_{0}\right)= \\
& \frac{1}{\left[4 \pi D\left(t-t_{0}\right)\right]^{3 / 2}} \exp \left(-\frac{\left(\boldsymbol{x}-\boldsymbol{x}_{0}-b F\left(t-t_{0}\right)\right)^{2}}{4 D\left(t-t_{0}\right)}\right) .
\end{aligned}
$$

The mean-square deviation calculated using the distributions (13) or (14) is given by the formula

$$
\left\langle\left(\boldsymbol{x}-\boldsymbol{x}_{0}\right)^{2}\right\rangle=2 D\left(t-t_{0}\right)+b^{2} F^{2}\left(t-t_{0}\right)^{2} .
$$

Using Eq. (15), one can estimate the effect of a constant external force on redistribution of particles initially concentrated in a small volume. First of all, it should be noted that the second term in the right-hand side of Eq. (15) is always positive and, hence, the external force always accelerates redistribution of particles over the volume. Further, taking into account the relation $b=D / k_{\mathrm{B}} T$, where $k_{\mathrm{B}}$ is the Boltzmann constant and $T$ - the medium temperature, and assuming that the second term has significant influence when it is comparable to the first one, the required magnitude of force can be estimated as $F \sim k_{\mathrm{B}} T / \sqrt{D\left(t-t_{0}\right)}$. It is seen from this formula that for inorganic molecules of the mass $m \approx 10^{-24} \mathrm{~g}$, diffusion coefficient $D \approx 10^{-6} \mathrm{~m}^{2} / \mathrm{s}$, and room temperature $300 \mathrm{~K}$, the characteristic time of particle redistribution should be $\delta t=t-t_{0} \approx 1 \mathrm{~s}$ for the influence of constant gravity to be significant. Obviously, these times should be several orders of magnitude less for organic molecules, which have much greater masses. Equation (15) can play an important role in an experimental investigation of external force influence on diffusion processes. This formula was first derived by Smoluchowski [14], who used the method different from reported here.

\section{Concentration nonlinearity of a liquid suspension of transparent microspheres}

In this section, starting from the one-dimensional Smoluchowski equation, we develop the theory of concentration nonlinearity of a liquid suspension of transparent microspheres under the action of a gradient force in the interference field of laser radiation. Although each of the suspension components (microspheres and liquid) does not exhibit nonlinearity, such an artificially created heterogeneous medium is a highly effective broadband nonlinear material for continuous laser radiation $[7,8]$. In the experimental study of four-wave mixing (FWM) of Ar-laser radiation $(\lambda=5145 \AA)$ in the suspension of latex microspheres of radius $R=1.17 \mu \mathrm{m}$ with concentration $N_{0}=6.5 \times 10^{10} \mathrm{~cm}^{-3}$, the optical Kerr coefficient was measured to be $n_{2}=3.6 \times 10^{-9} \mathrm{~cm}^{2} / \mathrm{W}$ [7], which is $10^{5}$ times greater than in carbon disulfide. In this case, the formation time $t_{F}$ and the decay time $t_{D}$ of the concentration gratings responsible for the FWM were $t_{F}=320 \mathrm{~ms}$ (at the pumping power of $\approx 100 \mathrm{~mW}$ ) and $t_{D}=200 \mathrm{~ms}$, respectively. The large value of nonlinear response time corresponds to the general regularity characteristic for nonlinear media - the linear dependence of the response time on the optical Kerr coefficient $n_{2}$ (see, e.g., [18]). The theory of FWM and stimulated concentration scattering in the aqueous suspension of transparent microspheres was developed in $[8,19]$ and [20], respectively. Stimulated Raman scattering in aqueous suspensions of diamond nanoparticles aggregates and monodisperse latex spheres was experimentally investigated in [21].

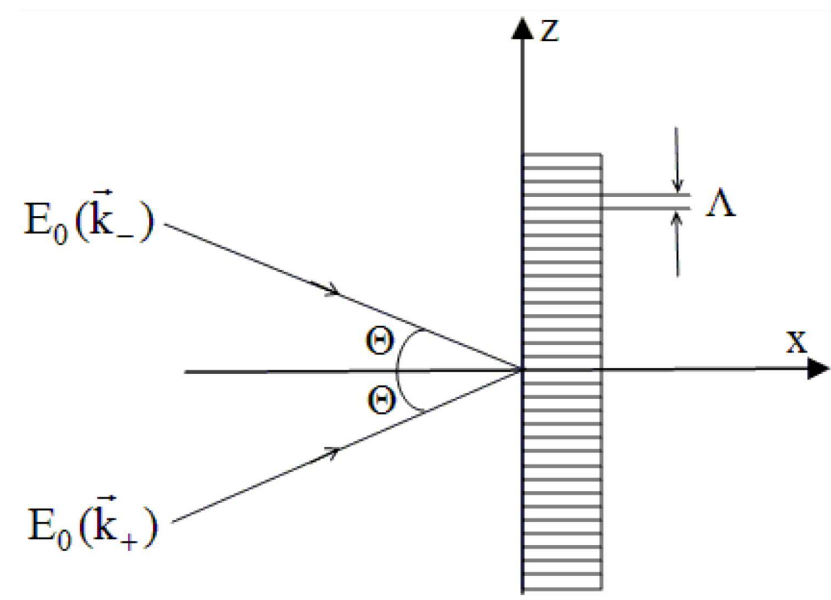

Fig. 1. Scheme of formation of the concentration grating.

We consider the concentration nonlinearity of a suspension of transparent microspheres under the action of a gradient force $F_{\nabla}$ in the field of two coherent waves of equal amplitude that converge at an angle $\Theta$ with the normal to the cell boundary (Fig. 1). Radiation intensity in the cell with the suspension has a form

$$
I(z, t)=I_{0}(t)\left[1+\cos \left(2 \pi \frac{z}{\Lambda}\right)\right],
$$

where $\Lambda=\pi / k \sin \Theta$ is the modulation period and $k$ the wave number. Intensity $I_{0}(t)$ is taken to be a square pulse of duration $\tau_{p}$ :

$$
I_{0}(t)=I_{0}\left[\Theta(t)-\Theta\left(t-\tau_{p}\right)\right],
$$


To describe the evolution of the microspheres concentration $N(z, t)$, we use the one-dimensional Smoluchowski equation

$$
\frac{\partial N}{\partial t}=D\left[\frac{\partial^{2} N}{\partial z^{2}}-\frac{1}{k_{\mathrm{B}} T}\left(N \frac{\partial F_{\nabla}}{\partial z}+F_{\nabla} \frac{\partial N}{\partial z}\right)\right] .
$$

In the Rayleigh-Gans approximation [22] and taking into account radiation inhomogeneity (16) in the microsphere volume $V=4 \pi R^{3} / 3$, the gradient force in Eq. (18) is given by $[8,23]$ :

$$
F_{\nabla}=2 \pi \frac{n}{c} \alpha I(t) \frac{1}{V} \int_{V} \nabla \cos \left(2 \pi \frac{z}{\Lambda}\right) \mathrm{d} V,
$$

where

$$
\alpha=R^{3} \frac{\bar{m}^{2}-1}{\bar{m}^{2}+2}
$$

is the microsphere polarizability and $\bar{m}=n_{0} / n$ is the ratio of the refractive index of the microsphere material $n_{0}$ to the refractive index of the liquid $n$ at the wavelength $\lambda$ (further, for definiteness, we assume $\alpha>0$ ). After integration in Eq. (19), we have

$$
\begin{aligned}
& F_{\nabla}=-4 \pi^{2} \frac{n}{c} \frac{\alpha}{\Lambda} I(t) U(\Omega) \sin \left(2 \pi \frac{z}{\Lambda}\right) \equiv \\
& \quad-F_{0} \sin \left(2 \pi \frac{z}{\Lambda}\right),
\end{aligned}
$$

where

$$
U(\Omega)=3 \sqrt{\frac{\pi}{2}} \Omega^{-3 / 2} J_{3 / 2}(\Omega)
$$

is a function, which describes radiation inhomogeneity in the microsphere volume; $J_{3 / 2}(\Omega)$ is the Bessel function of the order $3 / 2$, and $\Omega=2 \pi R / \Lambda(\Theta)$.

Figure 2 shows the angular dependence of the function $U(\Theta)$ for different values of microsphere radius $R$. It is seen that the inhomogeneity of radiation intensity in the microsphere volume governed by the relation $R / \Lambda(\Theta)$ results in decrease of the gradient force amplitude $F_{0}$ ( $\Theta=\pi / 2$ corresponds to counterpropagating waves, i.e. minimal modulation period $\Lambda=\pi / k)$. Since the function $U\left(\Omega \sim J_{3 / 2}(\Omega)\right.$ is sign-changing, $F_{0}(\Omega)$ changes sign at

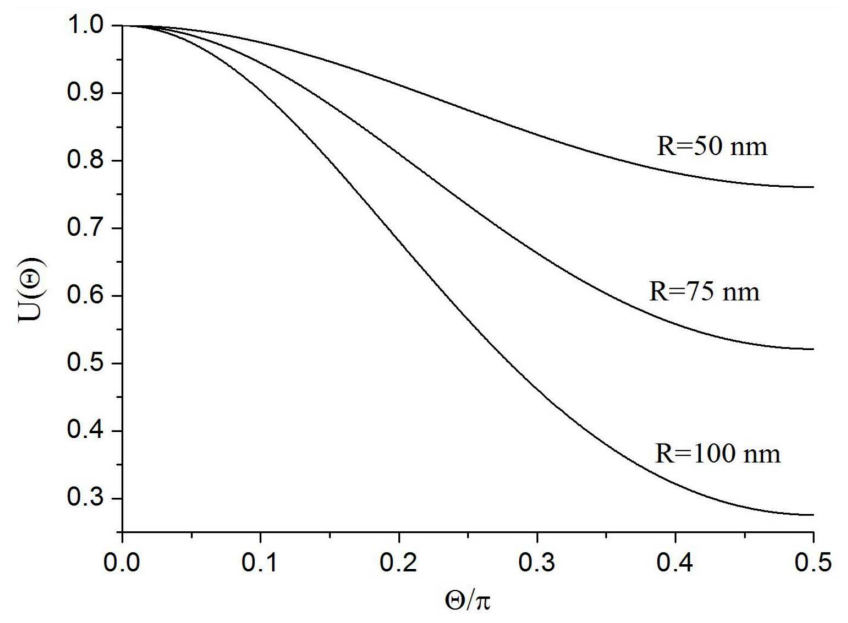

Fig. 2. The angular dependence of the function $U(\Theta)$ for different values of $R$. certain values of $\Omega$ and, hence, microspheres with $\alpha>0$ can be localized in the nodes of the field interference pattern. At $\Omega=\Omega_{i}$ (where $\Omega_{i}$ are the roots of the Bessel function, $i=1,2,3 \ldots)$, the gradient force $F_{0}\left(\Omega_{i}\right)=0$ irrespective of the microsphere position. The so-called "zero-force effect" [8] is due to the balance between force components acting on the corresponding elements of microsphere volume in the region where the particle overlaps two adjacent maxima (antinodes) of the interference pattern. In particular, for the first root of the Bessel function $J_{3 / 2}\left(\Omega_{1}=4.493\right)=0$, the "zero-force effect" is obtained at $R / \Lambda(\Theta)=0.3576$. In the region $\Omega_{1}<\Omega<\Omega_{2}$, $F_{0}<0$ and, consequently, the microsphere behaves like a particle with $\alpha<0$. Estimates show that the condition for the "zero-force effect" in the Rayleigh-Gans approximation used here can be realized for $|\bar{m}-1| \ll 1$.

Using the well-known relation

$$
J_{3 / 2}(\Omega)=\sqrt{\frac{2}{\pi \Omega}}\left(\frac{\sin \Omega}{\Omega}-\cos \Omega\right),
$$

assuming $\Omega \ll 1$ and leaving the first nonvanishing correction, one can show that

$$
U(\Omega) \approx 1-\frac{\Omega^{2}}{10} .
$$

It is obvious that the influence of the radiation inhomogeneity in the microsphere volume can be neglected only at $R / \Lambda \ll 1$.

Introducing the variable $\xi=2 \pi z / \Lambda, \mathrm{Eq}$. (18) can be rewritten for the function $\bar{N}(\xi, t)=N(\xi, t) / N_{0}$ as follows:

$$
\begin{aligned}
& \frac{\partial \bar{N}}{\partial t}= \\
& \quad\left(\frac{2 \pi}{\Lambda}\right)^{2} D_{0} \frac{\partial^{2} \bar{N}}{\partial \xi^{2}}+\frac{F_{0}}{3 \Lambda R \eta}\left(\bar{N} \cos \xi+\frac{\partial N}{\partial \xi} \sin \xi\right) .
\end{aligned}
$$

We seek a solution of Eq. (25) in the form of an expansion

$$
\bar{N}(\xi, t)=\sum_{\kappa=-\infty}^{\infty} \bar{N}_{\kappa}(t) \mathrm{e}^{\mathrm{i} \kappa \xi},
$$

where the Hermitian condition $\bar{N}_{\kappa}=\bar{N}_{-\kappa}^{*}$ is satisfied. Using Eq. (26), Eq. (25) is reduced to the infinite system of ordinary recurrent equations

$$
\frac{\mathrm{d} \bar{N}_{\kappa}}{\mathrm{d} t}=-\frac{\bar{N}_{\kappa}}{t_{\kappa}}+\kappa \frac{F_{0}}{6 \Lambda R \eta}\left(\bar{N}_{\kappa-1}-\bar{N}_{\kappa+1}\right)
$$

with the initial conditions $\bar{N}_{0}(t=0)=1$ and $\bar{N}_{\kappa \neq 0}(t=$ $0)=0$, where $t_{\kappa}=3 \Lambda^{2} R \eta / 2 \pi \kappa^{2} k_{\mathrm{B}} T$ is the diffusion decay time of the $\kappa$-th harmonic. It is obvious from Eq. (27) that even and odd harmonics are connected to each other. Since the amplitudes $\bar{N}_{\kappa}(t)$ are real, it suffices to consider only positive values of $\kappa(\kappa=1,2,3 \ldots)$.

Taking Eq. (26) into account, the solution of Eq. (25) is given by

$$
N(z, t)=N_{0}\left[1+2 \sum_{\kappa=1}^{\infty} \bar{N}_{\kappa}(t) \cos \left(2 \pi \kappa \frac{z}{\Lambda}\right)\right] .
$$

For numerical calculations, Eqs. (27) can be rewritten in a convenient form 


$$
\begin{aligned}
& \frac{\mathrm{d} \bar{N}_{\kappa}}{\mathrm{d} \tau}=-\kappa^{2} \bar{N}_{\kappa}+\kappa G_{0}\left(\bar{N}_{\kappa-1}-\bar{N}_{\kappa+1}\right), \\
& \kappa=1,2,3 \ldots,
\end{aligned}
$$

where $G_{0}=F_{0} \Lambda / 4 \pi k_{\mathrm{B}} T$ and $\tau=t / t_{1}$. Note that the coefficient $G_{0}$ does not depend on $\Lambda$ in the case $\Omega \ll 1$, when $F_{0} \sim 1 / \Lambda$.

In the steady state (at $\tau \gg 1$ ), Eqs. (29) lead to the system of algebraic equations

$$
\bar{N}_{\kappa}=\frac{G_{0}}{\kappa}\left(\bar{N}_{\kappa-1}-\bar{N}_{\kappa+1}\right) \text {. }
$$

In this case, the amplitudes of harmonics decrease with increasing $\kappa$ and simultaneously their coupling to neighboring harmonics gets weaker. For $G_{0}<1$, one can deduce from Eqs. (28) and (30) that

$$
N(z) \approx N_{0}\left[1+2 \sum_{\kappa=1}^{\infty} \frac{G_{0}^{\kappa}}{\kappa !} \cos \left(2 \pi \kappa \frac{z}{\Lambda}\right)\right] .
$$

Figure 3 shows the results of numerical calculation (from the system (29)) of the maximal concentration response $\bar{\Delta} \bar{N}(\tau)=2 \sum_{\kappa=1}^{\infty} \bar{N}_{\kappa}(\tau)$ in the antinodes of the interference pattern, i.e. when $\cos (2 \pi \kappa z /)=1$, for different values of the coefficient $G_{0}$ at $\Lambda(\Theta=\pi / 2)=\pi / \kappa$. Using these numerical solutions and the exponential approximation

$$
\bar{\Delta} \bar{N}(t)=\bar{\Delta} \bar{N}_{0}\left(1-\mathrm{e}^{-t / t_{\mathrm{F}}}\right),
$$

where $t_{F}$ is the formation time of the concentration grating, we obtain the dependence of $t_{F}$ on the coefficient $G_{0} \sim I_{0}$ (Fig. 4). Figure 4 shows that the time $t_{F}$ decreases with increasing intensity $I_{0}$ and, hence, $t_{F} \rightarrow t_{1}$ with decreasing $I_{0}$. It is also worth noting that in general, decrease of the formation time $t_{F}$ with the external force $\left(G_{0} \sim F_{0}\right)$ results from Eq. (15). This dependence was observed in experimental study of FWM in the water suspension of latex microspheres [7].

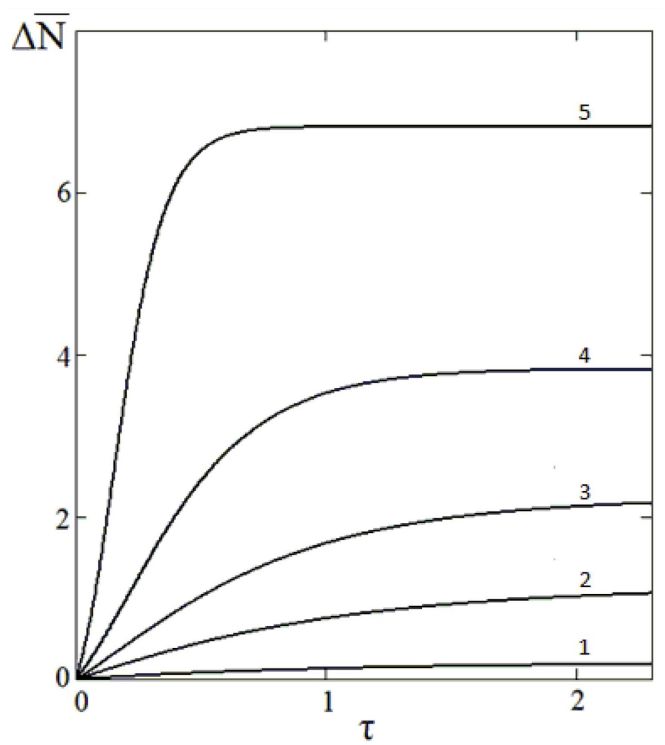

Fig. 3. Temporal dynamics of the concentration response at $\Lambda=\pi / \kappa$ for different values of $G_{0}: 1-0.1$, $2-0.5,3-1,4-2,5-5$.

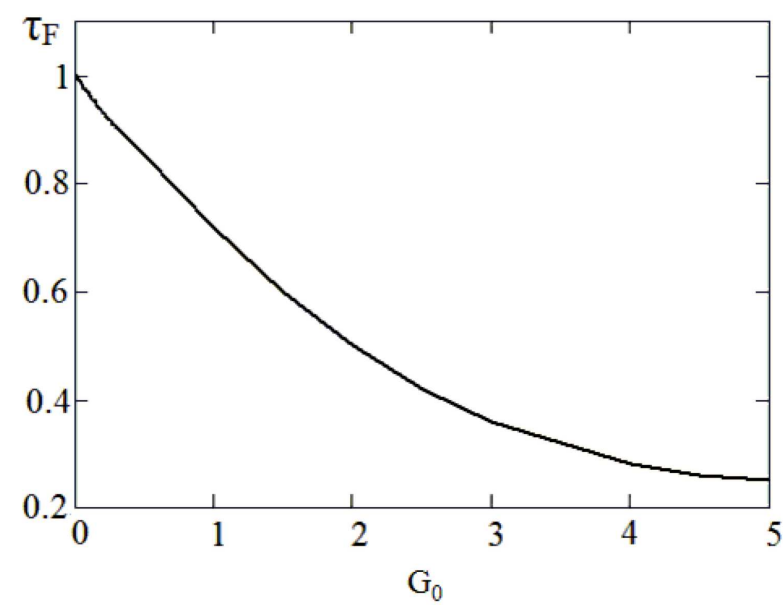

Fig. 4. Dependence of the normalized time $\tau_{F}=t_{F} / t_{1}$ on the coefficient $G_{0}$.

In the diffusion limit, $G_{0}<<1$ [8], when a single harmonic $\bar{N}_{1}(t)$ is enough to be considered, Eq. (29) results in

$$
\bar{N}_{1}(t)=G_{0}\left(1-\mathrm{e}^{-t / t_{0}}\right),
$$

where $t_{0}=t_{F}=t_{1}$. In this case, Eq. (29) has the form

$$
N(z, t)=N_{0}\left[1+2 G_{0}\left(1-\mathrm{e}^{-t / t_{0}}\right) \cos \left(2 \pi \frac{z}{\Lambda}\right)\right] .
$$

Estimates show that formula (34) is valid for the liquid suspension of latex microspheres with $R \approx 10^{-5} \mathrm{~cm}$ at $I_{0}<10^{2} \mathrm{~W} / \mathrm{cm}^{2}$. After "switching off" the radiation, relaxation of the main grating is described by

$$
\begin{aligned}
& \bar{N}_{1}(t)=2 G_{0}\left(1-\mathrm{e}^{-\tau_{p} / t_{0}}\right) \mathrm{e}^{-\left(t-\tau_{p}\right) / t_{0}} \\
& \text { for } t \geq \tau_{p} .
\end{aligned}
$$

To analyze the properties of the concentration grating (34), let us consider the Raman-Nath diffraction of a weak probe wave during grating excitation and relaxation. In this case, using Eq. (34) and the expression for the polarization of the diffracting weak wave $E_{c} \exp \left(\mathrm{i}\left(\boldsymbol{k}_{c} \cdot \boldsymbol{r}-\omega t\right)\right)$ :

$$
P_{c}=\left[\varepsilon_{0}+\alpha N(z, t)\right] E_{c} \mathrm{e}^{-\mathrm{i}\left(\omega t-\boldsymbol{k}_{c} \cdot \boldsymbol{r}\right)},
$$

one can find an equation for the amplitude $E_{c}$ :

$$
\cos \Theta_{c} \frac{\partial E_{c}}{\partial x}=\mathrm{i} \frac{\omega}{c} n_{2}^{0}\left(1-\mathrm{e}^{-t / t_{0}}\right) I_{0} \cos \left(2 \pi \frac{z}{\Lambda}\right) E_{c},
$$

where $\varepsilon_{0}$ is the dielectric permittivity of the liquid, $\Theta_{c}$ the angle between the vector $\boldsymbol{k}_{c}$ and the normal to the suspension layer,

$$
n_{2}^{0}=(2 \pi \alpha)^{2} N_{0} \frac{U(\Omega)}{c k_{\mathrm{B}} T}
$$

the optical Kerr coefficient, which generally depends not only on the suspension parameters $\left(\alpha, N_{0}, T\right)$, but also on the radiation modulation period $\Lambda$. Note that for $U(\Omega) \approx 1$, the coefficient $n_{2}^{0}>0$ regardless of the sign of $\alpha$ and is determined only by the parameters of the suspension, analogous to media with a cubic nonlinearity. For the latex microspheres in water at room temperature under the action of Ar-laser radiation $\left(\lambda_{0}=5145 \AA\right)$ and for the parameters of the experiment reported in 
Ref. [7] $\left(N_{0}=6.5 \times 10^{10} \mathrm{~cm}^{-3}, n_{0}=1.59, n=1.33\right.$, $\left.R=1.17 \times 10^{-5} \mathrm{~cm}, k_{\mathrm{B}} T=4.05 \times 10^{-14} \mathrm{erg}\right)$, we obtain from Eq. $(23)$ at $U(\Omega) \approx 1$ that $n_{2}^{0}=8.5 \times 10^{-10} \mathrm{~cm}^{2} / \mathrm{W}$. This value is $2.8 \times 10^{4}$ times greater than $n_{2}^{0}$ of carbon disulfide, which is $n_{2}^{0}\left(C S_{2}\right)=3 \times 10^{-14} \mathrm{~cm}^{2} / \mathrm{W}$. The value $n_{2}^{0}$ experimentally measured in the study of the concentration FWM is $n_{2}^{0}=3.6 \times 10^{-9} \mathrm{~cm}^{2} / \mathrm{W}$ [7]. This difference in the values of the Kerr coefficient is probably due to the use of the plane-wave approximation. For the parameters given above and for $\Lambda=\pi / k$ and $\eta=10^{-2} \mathrm{P}$, we have $t_{1}=0.5 \times 10^{-3} \mathrm{~s}$.

Using the solution of Eq. (37):

$$
E_{c}(L)=E_{c}(0) \cdot \mathrm{e}^{\mathrm{i} \delta(t) \cos (2 \pi z / L)}
$$

and the well-known relation

$$
\mathrm{e}^{\mathrm{i} \delta \sin (2 \pi z / L)}=\sum_{m=-\infty}^{\infty} J_{m}(\delta) \mathrm{e}^{\mathrm{i} m 2 \pi z / L},
$$

the efficiency of the $m$-th order diffraction can be written as (see, e.g., [24])

$$
\eta_{m}=J_{m}^{2}(\delta)
$$

where $\delta(t)=\frac{\omega}{c} n_{2}^{0}\left(1-\mathrm{e}^{-t / t_{0}}\right) I_{0}(t) L / \cos \Theta_{c}$ and $L$ is the thickness of the suspension layer. Direction of the diffraction maxima can be determined by the grating equation [18]:

$$
\cos \Theta_{m}=m \frac{\lambda}{\Lambda}+\cos \Theta_{c}, \quad m=0, \pm 1, \pm 2 \ldots
$$

Note that from the identity $\sum_{m=0}^{\infty} J_{m}^{2}=1$ follows the law of energy conservation for the diffracting wave.

Using the diffraction efficiency $\eta_{m}$, it is possible to experimentally measure the formation and decay time of the concentration grating as a function of the period $\Lambda$ and optical Kerr coefficient. In the case of large intensity $I_{0}$ (at $G_{0} \geq 1$ ), one can also measure the dependence of nonlinearity response time on the modulation period $\Lambda$ and radiation intensity.

\section{Conclusion}

In this paper, a number of problems were solved for the diffusion equation taking into account the presence of an external force (the Smoluchowski equation). Our goal is to develop an approach for theoretical estimate of the effect of external forces (predominantly gradient by nature) on the diffusion of particles in a liquid. The method of fundamental solution was used to solve these equations. The advantage of this method is that the fundamental solution has the direct physical meaning, namely, it is proportional to the distribution of diffusing particles. This feature is especially pronounced when solving the diffusion equation that takes into account the constant external force. As for the problem of the concentration response dynamics of particles under the influence of the gradient force induced by the interference field of two laser light beams, we have used the decomposition of the nonequilibrium concentration of particles by trigonometric functions. It is also shown that the solution of the diffusion equation taking into account the external force corresponds to the equilibrium (Boltzmann) distribution and describes the steady-state concentration grating.

\section{References}

[1] A. Ashkin, Sci. Am. 226, 63 (1972).

[2] A. Ashkin, J.M. Dziedzic, J.E. Bjorkholm, S. Chu, Opt. Lett. 11, 288 (1986).

[3] A. Ashkin, J.M. Dziedzic, T. Yamane, Nature 330 769 (1987).

[4] A. Ashkin, J.M. Dziedzic, Science 235, 1517 (1987).

[5] R.H. Austin, J.P. Brody, E.C. Cox, T. Duke, W. Volkmuth, Phys. Today 50, 32 (1997).

[6] Live Cell Imaging: A Laboratory Manual, Eds. R.D. Goldman, J.R. Swerdlow, D.L. Spector, 2nd ed., CSHL Press, Cold Spring Harbor 2010.

[7] P.W. Smith, A. Ashkin, W.J. Tomlinson, Opt. Lett. 6, 284 (1981)

[8] A.A. Afanas'ev, A.N. Rubinov, S.Yu. Mikhnevich, I.E. Ermolaev, J. Exp. Theor. Phys. 101, 389 (2005).

[9] L.D. Landau, E.M. Lifshitz, Fluid Mechanics, 2nd ed., Nauka, Moscow 1986; Butterworth-Heinemann, Oxford 1987.

[10] A.I. Akhiezer, S.V. Peletminskii, Methods of Statistical Physics, Nauka, Moscow 1977; Pergamon Press, Oxford 1981.

[11] E.M. Lifshitz, L.P. Pitaevskii, Physical Kinetics, Nauka, Moscow 1979; Pergamon Press, Oxford 1981.

[12] Yu.L. Klimontovich, Statistical Physics, Nauka, Moscow 1982; Harwood Academic Publ., New York 1986.

[13] A. Einstein, Ann. Phys. (Berlin) 322, 549 (1905).

[14] A. Einstein, M. Smoluchowski, Brownian Motion: A Collection of Papers, ONTI, Leningrad 1936 (in Russian).

[15] W. Ebeling, E. Gudowska-Nowak, I.M. Sokolov, Acta Phys. Pol. B 39, 1003 (2008).

[16] R.P. Feynman, A.R. Hibbs, Quantum Mechanics and Path Integrals, McGraw Hill, New York 1965; Mir, Moscow 1968.

[17] A.N. Vasiliev, Functional Methods in Quantum Field Theory and Statistical Physics, LGU, Leningrad 1976; Gordon and Breach, Amsterdam 1998.

[18] S.A. Akhmanov, S.Yu. Nikitin, Physical Optics, Clarendon Press, Oxford 1997; MGU, Moscow 1998.

[19] D. Rogovin, S.O. Sari, Phys. Rev. A 31, 2375 (1985).

[20] A.A. Afanas'ev, A.N. Rubinov, S.Yu. Mikhnevich, I.E. Ermolaev, Opt. Spectrosc. 102, 106 (2007).

[21] I.S. Burkhanov, S.V. Krivokhizha, L.L. Chaikov, Quantum Electron. 46, 548 (2016).

[22] H.C. van de Hulst, Light Scattering by Small Particles, Wiley, New York 1957; IL, Moscow 1961.

[23] P. Zemánek, A. Jonáš, M. Liška, J. Opt. Soc. Am. A19, 1025 (2002).

[24] A.L. Mikaelian, Optical Methods in Informatics, Nauka, Moscow 1990 (in Russian). 